\title{
The extinction and persistence of a stochastic SIR model
}

Chunyan $\mathrm{Ji}^{1,2^{*}}$ and Daqing Jiang 3,4

${ }^{*}$ Correspondence:
chunyanji80@hotmail.com
'School of Mathematics and
Statistics, Changshu Institute of
Technology, Changshu, Jiangsu
215500, P.R. China
${ }^{2}$ School of Mathematics and
Statistics, Northeast Normal
University, Changchun, Jilin 130024,
P.R. China
Full list of author information is
available at the end of the article

available at the end of the article

\begin{abstract}
The objective of this paper is to explore the long time behavior of a stochastic SIR model. We establish a threshold condition called the basic reproduction number under stochastic perturbation for persistence or extinction of the disease. Especially, some numerical simulations are applied to support our theoretical results.
\end{abstract}

MSC: 49K15; 60H10; 93E15

Keywords: a stochastic SIR model; extinction; persistence; threshold; basic reproduction number

\section{Introduction}

Epidemiology is the study of the spread of diseases with the objective to trace factors that are responsible for or contribute to their occurrence. Significant progress has been made in the theory and application by mathematical research [1-6].

Recently, we discussed the dynamic of a susceptible-infected-recovered (SIR) epidemic model with stochastic perturbation [7], that is,

$$
\left\{\begin{array}{l}
d S(t)=[\Lambda-\mu S(t)-\beta S(t) I(t)] d t-\sigma S(t) I(t) d B(t), \\
d I(t)=[\beta S(t) I(t)-(\mu+\gamma+\epsilon) I(t)] d t+\sigma S(t) I(t) d B(t), \\
d R(t)=[\gamma I(t)-\mu R(t)] d t,
\end{array}\right.
$$

where $S, I, R$, and $B(t)$ are the susceptible, the infective, the recovered, and a standard Brown motion, respectively. The parameters (all positive constants) have the following meaning: $\Lambda$ is the birth rate, $\mu$ is the death rate, $\beta$ is the average number of contacts per infective per day, $\gamma$ is the recovery rate, $\epsilon$ is the death rate of infective caused by the disease, and $\sigma^{2}$ is the intensity of the white noise. We investigated the persistence and extinction of the disease, and so we gave the threshold of this stochastic model, which is affected by white noise and is less than the basic reproduction number of the corresponding deterministic system. However, considering stochastic perturbations to have different patterns, it is interesting to investigate whether the threshold of this model with other stochastic perturbations has a relation with white noise [8-10].

(c) The Author(s) 2017. This article is distributed under the terms of the Creative Commons Attribution 4.0 International License (http://creativecommons.org/licenses/by/4.0/), which permits unrestricted use, distribution, and reproduction in any medium, provided you give appropriate credit to the original author(s) and the source, provide a link to the Creative Commons license, and indicate if changes were made. 
We devote this paper to the study of the following stochastic system:

$$
\left\{\begin{array}{l}
d S(t)=[\Lambda-\mu S(t)-\beta S(t) I(t)] d t+\sigma_{1} S(t) d B_{1}(t), \\
d I(t)=[\beta S(t) I(t)-(\mu+\gamma+\epsilon) I(t)] d t+\sigma_{2} I(t) d B_{2}(t), \\
d R(t)=[\gamma I(t)-\mu R(t)] d t+\sigma_{3} R(t) d B_{3}(t),
\end{array}\right.
$$

where $B_{1}(t), B_{2}(t), B_{3}(t)$ are mutually independent Brown motions with intensities $\sigma_{1}^{2}, \sigma_{2}^{2}, \sigma_{3}^{2}$, respectively. For any initial value $(S(0), I(0), R(0)) \in R_{+}^{3}$, there is a solution $(S(t), I(t), R(t)) \in R_{+}^{3}$ of system (1.2) (see [11]). In this paper, we also intend to give the basic reproduction number of this system through discussing the long time behavior of the model, which will be given in the next section. In Section 3, we illustrate results through simulations. At the end, a discussion is given to conclude this paper.

Throughout this paper, unless otherwise specified, let $\left(\Omega, \mathcal{F},\left\{\mathcal{F}_{t}\right\}_{t \geq 0}, P\right)$ be a complete probability space with a filtration $\left\{\mathcal{F}_{t}\right\}_{t \geq 0}$ satisfying the usual conditions (i.e. it is right continuous and $\mathscr{F}_{0}$ contains all P-null sets), and let $B(t)$ be a scalar Brownian motion defined on the probability space.

\section{The long time behavior of system (1.2)}

In this section, we deduce when the disease will die out and when the disease will prevail. And so the basic reproduction number of system (1.2) is given. As the proof in [12], we can conclude to the following lemmas.

Lemma 2.1 Let $(S(t), I(t), R(t))$ be the solution of system (1.2) with any initial value $(S(0), I(0), R(0)) \in R_{+}^{3}$. Then

$$
\lim _{t \rightarrow \infty} \frac{S(t)+I(t)+R(t)}{t}=0 \quad \text { a.s. }
$$

Remark 2.1 In fact, together with the positivity of the solution and (2.1), we have

$$
\lim _{t \rightarrow \infty} \frac{S(t)}{t}=0, \quad \lim _{t \rightarrow \infty} \frac{I(t)}{t}=0, \quad \lim _{t \rightarrow \infty} \frac{R(t)}{t}=0 \quad \text { a.s. }
$$

Lemma 2.2 Assume $\mu>\frac{1}{2}\left(\sigma_{1}^{2} \vee \sigma_{2}^{2} \vee \sigma_{3}^{2}\right)$. Let $(S(t), I(t), R(t))$ be the solution of system (1.2) with any initial value $(S(0), I(0), R(0)) \in R_{+}^{3}$, then

$$
\begin{aligned}
& \lim _{t \rightarrow \infty} \frac{\int_{0}^{t} S(r) d B_{1}(r)}{t}=0, \quad \lim _{t \rightarrow \infty} \frac{\int_{0}^{t} I(r) d B_{2}(r)}{t}=0, \\
& \lim _{t \rightarrow \infty} \frac{\int_{0}^{t} R(r) d B_{3}(r)}{t}=0 \quad \text { a.s. }
\end{aligned}
$$

Let

$$
\tilde{R}_{0}=\frac{\beta \Lambda}{\mu\left(\mu+\gamma+\epsilon+\frac{\sigma_{2}^{2}}{2}\right)}=R_{0}-\frac{\beta \Lambda}{2 \mu(\mu+\gamma+\epsilon)\left(\mu+\gamma+\epsilon+\frac{\sigma_{2}^{2}}{2}\right)} \sigma_{2}^{2},
$$

where $R_{0}=\frac{\beta \Lambda}{\mu(\mu+\gamma+\epsilon)}$ is the basic reproduction of the corresponding deterministic system [13]. Based on these two lemmas, we give mainly the results in this section. 
Theorem 2.1 Assume $\mu>\frac{1}{2}\left(\sigma_{1}^{2} \vee \sigma_{2}^{2} \vee \sigma_{3}^{2}\right)$. Let $(S(t), I(t), R(t))$ be the solution of system (1.2) with any initial value $(S(0), I(0), R(0)) \in R_{+}^{3}$. If $\tilde{R}_{0}<1$, then

$$
\limsup _{t \rightarrow \infty} \frac{\log I(t)}{t} \leq\left(\mu+\gamma+\epsilon+\frac{\sigma_{2}^{2}}{2}\right)\left(\tilde{R}_{0}-1\right)<0 \quad \text { a.s. }
$$

namely, $I(t)$ tends to zero exponentially a.s. In other words, the disease dies out with probability one.

Proof Note that

$$
\begin{aligned}
& \frac{S(t)-S(0)}{t}=\Lambda-\mu \frac{\int_{0}^{t} S(s) d s}{t}-\beta \frac{\int_{0}^{t} S(s) I(s) d s}{t}+\sigma_{1} \frac{\int_{0}^{t} S(s) d B_{1}(s)}{t} \\
& \frac{I(t)-I(0)}{t}=\beta \frac{\int_{0}^{t} S(s) I(s) d s}{t}-(\mu+\gamma+\epsilon) \frac{\int_{0}^{t} I(s) d s}{t}+\sigma_{2} \frac{\int_{0}^{t} I(s) d B_{2}(s)}{t} \\
& \frac{R(t)-R(0)}{t}=\gamma \frac{\int_{0}^{t} I(s) d s}{t}-\mu \frac{\int_{0}^{t} R(s) d s}{t}+\sigma_{3} \frac{\int_{0}^{t} R(s) d B_{3}(s)}{t}
\end{aligned}
$$

then

$$
\begin{aligned}
& \mu \frac{\int_{0}^{t} S(s) d s}{t}+(\mu+\gamma+\epsilon) \frac{\int_{0}^{t} I(s) d s}{t} \\
& \quad=\Lambda-\frac{S(t)+I(t)}{t}+\frac{S(0)+I(0)}{t}+\sigma_{1} \frac{\int_{0}^{t} S(s) d B_{1}(s)}{t}+\sigma_{2} \frac{\int_{0}^{t} I(s) d B_{2}(s)}{t} \\
& \quad:=\Lambda+\varphi(t)
\end{aligned}
$$

where $\varphi(t)$ has the property that

$$
\lim _{t \rightarrow \infty} \varphi(t)=0
$$

according to (2.2) and (2.3). Then

$$
\lim _{t \rightarrow \infty} \frac{\mu \int_{0}^{t} S(s) d s+(\mu+\gamma+\epsilon) \int_{0}^{t} I(s) d s}{t}=\Lambda .
$$

In addition,

$$
\begin{aligned}
\log I(t)= & \log I(0)+\beta \int_{0}^{t} S(s) d s-\left(\mu+\gamma+\epsilon+\frac{\sigma_{2}^{2}}{2}\right) t+\sigma_{2} B_{2}(t) \\
= & \log I(0)+\frac{\beta \Lambda}{\mu} t-\frac{\beta(\mu+\gamma+\epsilon)}{\mu} \int_{0}^{t} I(s) d s+\frac{\beta}{\mu} \varphi(t) t-\left(\mu+\gamma+\epsilon+\frac{\sigma_{2}^{2}}{2}\right) t \\
& +\sigma_{2} B_{2}(t) \\
= & \left.\frac{\beta \Lambda}{\mu}-\left(\mu+\gamma+\epsilon+\frac{\sigma_{2}^{2}}{2}\right)\right] t-\frac{\beta(\mu+\gamma+\epsilon)}{\mu} \int_{0}^{t} I(s) d s+\log I(0)+\frac{\beta}{\mu} \varphi(t) t \\
& +\sigma_{2} B_{2}(t) \\
\leq & {\left[\frac{\beta \Lambda}{\mu}-\left(\mu+\gamma+\epsilon+\frac{\sigma_{2}^{2}}{2}\right)\right] t+\log I(0)+\frac{\beta}{\mu} \varphi(t) t+\sigma_{2} B_{2}(t) }
\end{aligned}
$$


and

$$
\lim _{t \rightarrow \infty} \frac{1}{t}\left[\log I(0)+\frac{\beta}{\mu} \varphi(t) t+\sigma_{2} B_{2}(t)\right]=0 \quad \text { a.s. }
$$

by (2.5) and the property of the Brown motion. If $\tilde{R}_{0}<1$, from (2.7), we have

$$
\limsup _{t \rightarrow \infty} \frac{\log I(t)}{t} \leq \frac{\beta \Lambda}{\mu}-\left(\mu+\gamma+\epsilon+\frac{\sigma_{2}^{2}}{2}\right)=\left(\mu+\gamma+\epsilon+\frac{\sigma_{2}^{2}}{2}\right)\left(\tilde{R}_{0}-1\right)<0
$$

as required.

Theorem 2.2 Assume $\mu>\frac{1}{2}\left(\sigma_{1}^{2} \vee \sigma_{2}^{2} \vee \sigma_{3}^{2}\right)$. Let $(S(t), I(t), R(t))$ be the solution of system (1.2) with any initial value $(S(0), I(0), R(0)) \in R_{+}^{3}$. If $\tilde{R}_{0}<1$, then

$$
\lim _{t \rightarrow \infty} \frac{1}{t} \int_{0}^{t} S(r) d r=\frac{\Lambda}{\mu}, \quad \lim _{t \rightarrow \infty} I(t)=0, \quad \lim _{t \rightarrow \infty} R(t)=0 \quad \text { a.s. }
$$

Proof We have $\tilde{R}_{0}<1$, let $-\kappa=\left(\mu+\gamma+\epsilon+\frac{\sigma_{2}^{2}}{2}\right)\left(\tilde{R}_{0}-1\right)<0$. It follows from the result of Theorem 2.1 that, for arbitrary small constant $0<\xi<\min \{1 / 2, \kappa / 2\}$, there exist a constant $T=T(\omega)$ and a set $\Omega_{\xi}$ such that $P\left(\Omega_{\xi}\right) \geq 1-\xi$ and for $t \geq T, \omega \in \Omega_{\xi}, \log I(t) \leq-\frac{\kappa}{2} t$, and so $I(t) \leq e^{-\kappa t / 2}$. Therefore $\limsup _{t \rightarrow \infty} I(t) \leq 0$ a.s., which together with the positivity of the solution implies

$$
\lim _{t \rightarrow \infty} I(t)=0 \quad \text { a.s. }
$$

Therefore, from (2.6), (2.8), and the third equations of (1.2), it is easy to obtain

$$
\lim _{t \rightarrow \infty} \frac{1}{t} \int_{0}^{t} S(s) d s=\frac{\Lambda}{\mu}, \quad \lim _{t \rightarrow \infty} R(t)=0 \quad \text { a.s. }
$$

The proof of this result is completed.

In the previous, we show the extinction of the disease. Now, we investigate conditions for the prevalence of the disease.

Theorem 2.3 Assume $\mu>\frac{1}{2}\left(\sigma_{1}^{2} \vee \sigma_{2}^{2} \vee \sigma_{3}^{2}\right)$. Let $(S(t), I(t), R(t))$ be the solution of system (1.2) with any initial value $(S(0), I(0), R(0)) \in R_{+}^{3}$. If $\tilde{R}_{0}>1$, then

$$
\begin{aligned}
& \lim _{t \rightarrow \infty} \frac{1}{t} \int_{0}^{t} S(s) d s=\frac{\Lambda}{\mu \tilde{R}_{0}} \quad \text { a.s., } \\
& \lim _{t \rightarrow \infty} \frac{1}{t} \int_{0}^{t} I(s) d s=\frac{\mu\left(\mu+\gamma+\epsilon+\frac{\sigma_{2}^{2}}{2}\right)}{\beta(\mu+\gamma+\epsilon)}\left(\tilde{R}_{0}-1\right) \quad \text { a.s., } \\
& \lim _{t \rightarrow \infty} \frac{1}{t} \int_{0}^{t} R(s) d s=\frac{\gamma\left(\mu+\gamma+\epsilon+\frac{\sigma_{2}^{2}}{2}\right)}{\beta(\mu+\gamma+\epsilon)}\left(\tilde{R}_{0}-1\right) \quad \text { a.s. }
\end{aligned}
$$


Proof If $\tilde{R}_{0}>1$, then from the last equality of (2.7) and Lemmas 5.1, 5.2 in [7], we have

$$
\lim _{t \rightarrow \infty} \frac{1}{t} \int_{0}^{t} I(s) d s=\frac{\frac{\beta \Lambda}{\mu}-\left(\mu+\gamma+\epsilon+\frac{\sigma_{2}^{2}}{2}\right)}{\frac{\beta(\mu+\gamma+\epsilon)}{\mu}}=\frac{\mu\left(\mu+\gamma+\epsilon+\frac{\sigma_{2}^{2}}{2}\right)}{\mu+\gamma+\epsilon}\left(\tilde{R}_{0}-1\right) \quad \text { a.s. }
$$

Together with (2.6), this yields

$$
\lim _{t \rightarrow \infty} \frac{1}{t} \int_{0}^{t} S(s) d s=\frac{\Lambda}{\mu}-\frac{\left(\mu+\gamma+\epsilon+\frac{\sigma_{2}^{2}}{2}\right)}{\beta}\left(\tilde{R}_{0}-1\right)=\frac{\Lambda}{\mu \tilde{R}_{0}} \quad \text { a.s. }
$$

Moreover, from the third equation of system (1.2), we obtain

$$
\frac{R(t)-R(0)}{t}=\frac{\gamma}{t} \int_{0}^{t} I(s) d s-\frac{\mu}{t} \int_{0}^{t} R(s) d s+\frac{\sigma_{3}}{t} \int_{0}^{t} R(s) d B_{3}(s),
$$

which together with (2.3) and (2.9) implies

$$
\lim _{t \rightarrow \infty} \frac{1}{t} \int_{0}^{t} R(s) d s=\frac{\gamma\left(\mu+\gamma+\epsilon+\frac{\sigma_{2}^{2}}{2}\right)}{\beta(\mu+\gamma+\epsilon)}\left(\tilde{R}_{0}-1\right) \quad \text { a.s. }
$$

Remark 2.2 Although there is no endemic equilibrium of system (1.2), Theorem 2.3 tells us the solution of it tends to a point in time on average whose value is less than the value of $\left(S^{*}, I^{*}, R^{*}\right)$ [13], and we consider it is stable in time on average.

Furthermore, from the results of Theorem 2.2 and Theorem 2.3, we see that the value of $\tilde{R}_{0}$ mainly determines the extinction and persistence of the disease. As deterministic epidemic models, $\tilde{R}_{0}$ can be called the basic reproduction of the stochastic epidemic system (1.2).

\section{Simulations}

In this section, using the method mentioned in [14], we give simulations to support our results. The discretized equation is

$$
\left\{\begin{array}{l}
S_{k+1}=S_{k}+\left(\Lambda-\mu S_{k}-\beta S_{k} I_{k}\right) \Delta t+\sigma_{1} \epsilon_{1, k} \sqrt{\Delta t} S_{k} \\
I_{k+1}=I_{k}+\left[\beta S_{k} I_{k}-(\mu+\gamma+\epsilon) I_{k}\right] \Delta t+\sigma_{2} \epsilon_{2, k} \sqrt{\Delta t} I_{k} \\
R_{k+1}=R_{k}+\left(\gamma I_{k}-\mu R_{k}\right) \Delta t+\sigma_{3} \epsilon_{2, k} \sqrt{\Delta t} R_{k}
\end{array}\right.
$$

Let $(S(0), I(0), R(0))=(2,5,3), \Lambda=100, \beta=2, \mu=10, \gamma=4, \epsilon=1, \Delta=0.001$. In this situation, $R_{0}=\frac{4}{3}>1$, then the solution of the corresponding deterministic system will tend to $\left(S^{*}, I^{*}, R^{*}\right)=\left(\frac{15}{2}, \frac{5}{3}, \frac{2}{3}\right)$ and the disease will prevail (see the black real lines in Figures 1 and 2). Choosing different values of $\sigma_{1}, \sigma_{2}, \sigma_{3}$, we get two cases.

Case 1 Assume $\sigma_{1}=0.5, \sigma_{2}=4, \sigma_{3}=0.2$, then $\mu>\frac{1}{2}\left(\sigma_{1}^{2} \vee \sigma_{2}^{2} \vee \sigma_{3}^{2}\right)$ and $\tilde{R}_{0}=\frac{20}{23}<1$. As expected, the infected population $I(t)$ will tend to 0 very quickly and the disease will die out. The susceptible population $S(t)$ will tend to $\frac{\Lambda}{\mu}=10$ in time average (see the second picture in Figure 1).

Case 2 Let $\sigma_{1}=0.5, \sigma_{2}=1.5, \sigma_{3}=0.2$ such that $\mu>\frac{1}{2}\left(\sigma_{1}^{2} \vee \sigma_{2}^{2} \vee \sigma_{3}^{2}\right)$ and $\tilde{R}_{0}=\frac{160}{129}>1$. As Theorem 2.3 said, the disease will prevail and the solution is fluctuating around the endemic equilibrium $\left(S^{*}, I^{*}, R^{*}\right)$ of the corresponding deterministic system (see the upper three pictures in Figure 2). Furthermore, we show that the susceptible, infected and re- 

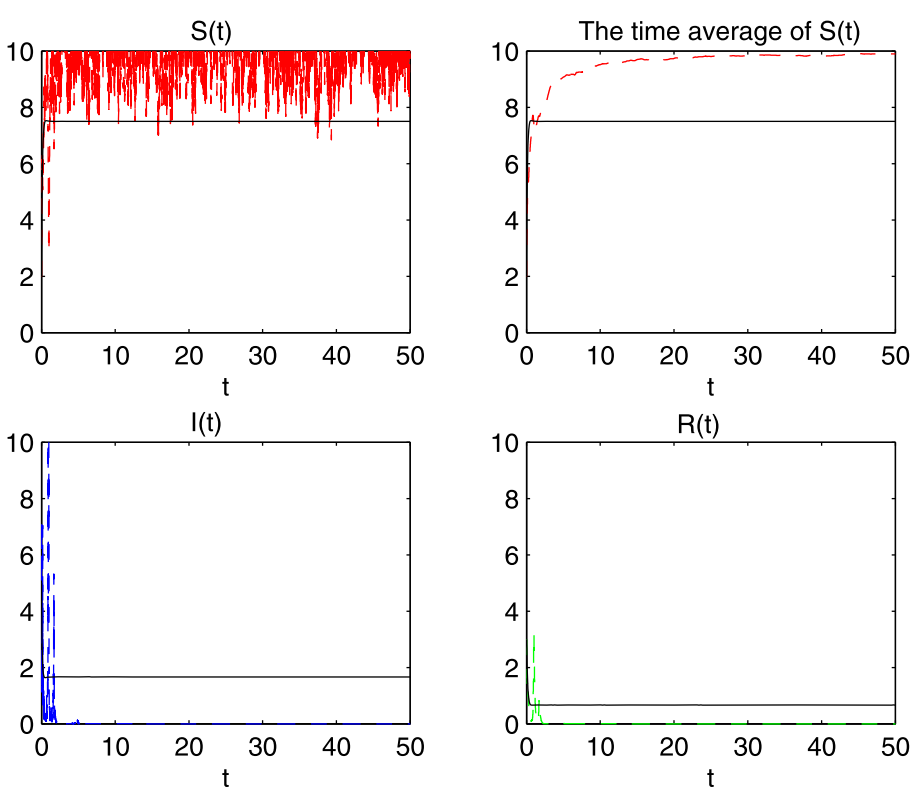

Figure 1 Simulation of the path $S(t), \frac{1}{t} \int_{0}^{t} S(s) d s, I(t)$ and $R(t)$ for the stochastic system (1.2) and the corresponding deterministic system with $R_{0}>1$ and $\tilde{R}_{0}<1$.
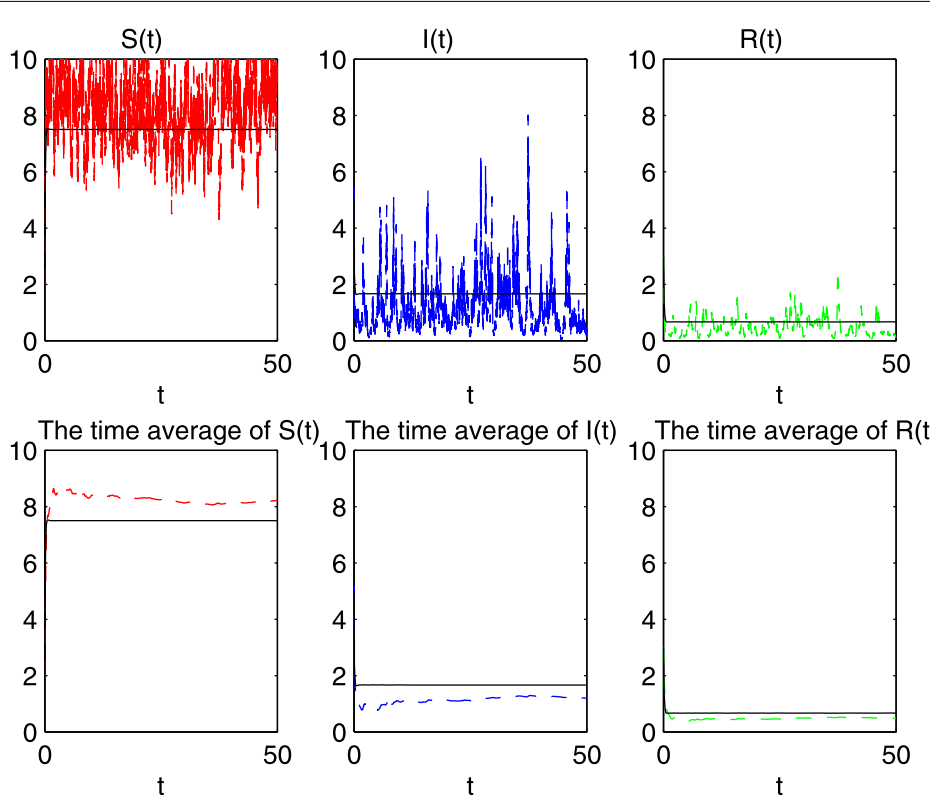

Figure 2 Simulation of the path $S(t), I(t), R(t)$, and $\frac{1}{t} \int_{0}^{t} S(s) d s, \frac{1}{t} \int_{0}^{t} I(s) d s, \frac{1}{t} \int_{0}^{t} R(s) d s$ for the stochastic system (1.2) and the corresponding deterministic system with $R_{0}>1$ and $\tilde{R}_{0}>1$.

covered population will tend to a positive value in time on average, respectively, in detail,

$$
\lim _{t \rightarrow \infty} \frac{1}{t} \int_{0}^{t} S(s) d s=\frac{129}{16}, \quad \lim _{t \rightarrow \infty} \frac{1}{t} \int_{0}^{t} I(s) d s=\frac{31}{24}, \quad \lim _{t \rightarrow \infty} \frac{1}{t} \int_{0}^{t} R(s) d s=\frac{31}{60} \quad \text { a.s. }
$$

The other three pictures in Figure 2 illustrate this. 


\section{Discussions}

In this paper, by qualitative analysis we have studied the global behavior of an SIR model with stochastic perturbation. As the deterministic epidemic models, we give a basic reproduction number $\tilde{R}_{0}$ of system (1.2), which is also less than the value of the basic reproduction number in the corresponding deterministic system. From the discussion, we can think white noise can make the value of the basic reproduction number lower, and so it is beneficial for controlling the disease.

In fact, for a stochastic SIRS epidemic model,

$$
\left\{\begin{array}{l}
d S(t)=[\Lambda-\mu S(t)-\beta S(t) I(t)+\rho R(t)] d t+\sigma_{1} S(t) d B_{1}(t) \\
d I(t)=[\beta S(t) I(t)-(\mu+\gamma+\epsilon) I(t)] d t+\sigma_{2} I(t) d B_{2}(t), \\
d R(t)=[\gamma I(t)-\mu R(t)-\rho R(t)] d t+\sigma_{3} R(t) d B_{3}(t)
\end{array}\right.
$$

we can obtain the following properties through a similar analysis.

- If $\mu>\frac{1}{2}\left(\sigma_{1}^{2} \vee \sigma_{2}^{2} \vee \sigma_{3}^{2}\right)$ and $\tilde{R}_{0}<1$, then

$$
\begin{aligned}
& \lim _{t \rightarrow \infty} \frac{\log I(t)}{t}=\left(\mu+\gamma+\epsilon+\frac{\sigma_{2}^{2}}{2}\right)\left(\tilde{R}_{0}-1\right)<0 \quad \text { a.s. } \\
& \lim _{t \rightarrow \infty} \frac{1}{t} \int_{0}^{t} S(r) d r=\frac{\Lambda}{\mu}, \quad \lim _{t \rightarrow \infty} I(t)=0, \quad \lim _{t \rightarrow \infty} R(t)=0 \quad \text { a.s. }
\end{aligned}
$$

- If $\mu>\frac{1}{2}\left(\sigma_{1}^{2} \vee \sigma_{2}^{2} \vee \sigma_{3}^{2}\right)$ and $\tilde{R}_{0}>1$, then

$$
\begin{aligned}
& \lim _{t \rightarrow \infty} \frac{1}{t} \int_{0}^{t} S(s) d s=\frac{\Lambda}{\mu \tilde{R}_{0}} \quad \text { a.s., } \\
& \lim _{t \rightarrow \infty} \frac{1}{t} \int_{0}^{t} I(s) d s=\frac{\mu(\mu+\rho)\left(\mu+\gamma+\epsilon+\frac{\sigma_{2}^{2}}{2}\right)}{\beta[(\mu+\rho)(\mu+\epsilon)+\mu \gamma]}\left(\tilde{R}_{0}-1\right) \quad \text { a.s., } \\
& \lim _{t \rightarrow \infty} \frac{1}{t} \int_{0}^{t} R(s) d s=\frac{\mu \gamma\left(\mu+\gamma+\epsilon+\frac{\sigma_{2}^{2}}{2}\right)}{\beta[(\mu+\rho)(\mu+\epsilon)+\mu \gamma]}\left(\tilde{R}_{0}-1\right) \quad \text { a.s. }
\end{aligned}
$$

\section{Competing interests}

The authors declare that they have no competing interests.

\section{Authors' contributions}

CJ contributed to Sections 1, 2, 3, and 4. Daqing Jiang contributed to Sections 2 and 4.

\section{Author details}

${ }^{1}$ School of Mathematics and Statistics, Changshu Institute of Technology, Changshu, Jiangsu 215500, P.R. China. ${ }^{2}$ School of Mathematics and Statistics, Northeast Normal University, Changchun, Jilin 130024, P.R. China. ${ }^{3}$ College of Science, China University of Petroleum (East China), Qingdao, 266580, P.R. China. ${ }^{4}$ Nonlinear Analysis and Applied Mathematics (NAAM)-Research Group, Department of Mathematics, King Abdulaziz University, Jeddah, Saudi Arabia.

\section{Acknowledgements}

The work was supported by the National Natural Science Foundation of China (No. 11601043), the National Science Foundation for Post-doctoral Scientists of China (No. 2016M590243) and sponsored by Qing Lan Project of Jiangsu Province (2016).

Received: 29 July 2016 Accepted: 26 December 2016 Published online: 26 January 2017

\section{References}

1. Anderson, RM, May, RM: Population biology of infectious diseases, part I. Nature 280, 361-367 (1979)

2. Beretta, E, Takeuchi, Y: Convergence results in SIR epidemic model with varying population sizes. Nonlinear Anal. 28, 1909-1921 (1997) 
3. Beretta, E, Hara, T, Ma, W, Takeuchi, Y: Global asymptotic stability of an SIR epidemic model with distributed time delay. Nonlinear Anal. 47, 4107-4115 (2001)

4. Franceschetti, A, Pugliese, A: Threshold behaviour of a SIR epidemic model with age structure and immigration. J. Math. Biol. 57, 1-27 (2008)

5. Jana, S, Haldar, P, Kar, TK: Mathematical analysis of an epidemic model with isolation and optimal controls. Int. J. Comput. Math. 1-22 (2016)

6. Zaman, G, Kang, YH, Jung, IH: Stability analysis and optimal vaccination of an SIR epidemic model. Biosystems 93 , 240-249 (2008)

7. Ji, CY, Jiang, DQ: Threshold behaviour of a stochastic SIR model. Appl. Math. Model. 38, 5067-5079 (2014)

8. Lin, YG, Jiang, DQ, Xia, PY: Long-time behavior of a stochastic SIR model. Appl. Math. Comput. 236, 1-9 (2014)

9. Zhao, DL: Study on the threshold of a stochastic SIR epidemic model and its extensions. Commun. Nonlinear Sci. Numer. Simul. 38, 172-177 (2016)

10. Zhou, YL, Zhang, WG: Threshold of a stochastic SIR epidemic model with Lévy jumps. Physica A 446, 204-216 (2016)

11. Ji, CY, Jiang, DQ, Yang, QS, Shi, NZ: Dynamics of a multigroup SIR epidemic model with stochastic perturbation. Automatica 48, 121-131 (2012)

12. Zhao, YN, Jiang, DQ: The threshold of a stochastic SIS epidemic model with vaccination. Appl. Math. Comput. 243, 718-727 (2014)

13. Hethcote, HW: Qualitative analyses of communicable disease models. Math. Biosci. 7, 335-356 (1976)

14. Higham, DJ: An algorithmic introduction to numerical simulation of stochastic differential equations. SIAM Rev. 43 , 525-546 (2001)

\section{Submit your manuscript to a SpringerOpen ${ }^{\circ}$ journal and benefit from:}

- Convenient online submission

Rigorous peer review

- Immediate publication on acceptance

- Open access: articles freely available online

- High visibility within the field

- Retaining the copyright to your article 